Boletín de la Sociedad Geológica Mexicana

VOLUMEN 68, NÚM. 2, 2016, P. 313-321

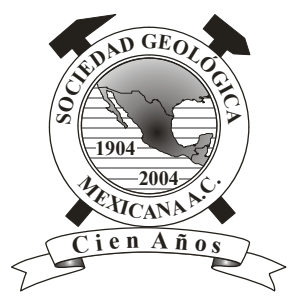

\title{
Braquiópodos discínidos (Lingulida, Discinoidea) de la Formación Ixtaltepec, Carbonífero del área de Santiago Ixtaltepec, Oaxaca
}

\author{
Miguel Angel Torres-Martínez ${ }^{1, *}$, Francisco Sour-Tovar ${ }^{2}$ \\ ${ }^{1}$ Instituto de Geología, Departamento de Paleontología, Universidad Nacional Autónoma de México, Ciudad Universitaria, 04510 , \\ CDMX, México. \\ ${ }^{2}$ Museo de Paleontología, Departamento de Biología Evolutiva, Facultad de Ciencias, Universidad Nacional Autónoma de México, \\ Ciudad Universitaria, 04510 CDMX, México. \\ *miguelatm@geologia.unam.mx
}

\section{Resumen}

Se describen cuatro especies de braquiópodos discínidos del género Orbiculoidea, presentes en diferentes niveles de la sección tipo de la Formación Ixtaltepec, Arroyo las Pulgas, Municipio de Nochixtlán, Oaxaca. Orbiculoidea caneyana y Orbiculoidea sp. se encontraron en niveles basales de la formación, en estratos de lutita de edad chesteriana (Misisípico Tardío). Orbiculoidea missouriensis y Orbiculoidea capuliformis se hallan en niveles estratigráficos superiores, también de lutita, de edad morrowano-desmoinesiana (Pensilvánico Medio), corroborando la edad de las rocas portadoras. Los reportes de Orbiculoidea caneyana y O. capuliformis son los primeros que se hacen para México y al igual que el registro de O. missouriensis remarcan las relaciones paleobiogeográficas de las faunas carboníferas de Nochixtlán con faunas coetáneas que se han descrito para la región centro-este de los Estados Unidos.

Palabras clave: Braquiópodos, Orbiculoidea, Carbonífero, Chesteriano, Morrowano-Desmoinesiano, México.

\begin{abstract}
Four species of discinid brachiopods of the genus Orbiculoidea present at different levels of the Ixtaltepec Formation, type section Arroyo las Pulgas, Nochixtlán Municipality, Oaxaca are described. Orbiculoidea caneyana and Orbiculoidea sp. were found in basal levels of the formation, in shale strata of chesterian age (Late Mississippian). The presence of Orbiculoidea missouriensis and Orbiculoidea capuliformis in upper stratigraphic levels, and also in argillaceous rocks of morrowan-desmoinesian age (Middle Pennsylvanian), corroborate the age of the fossil-bearing rocks. These are the first records of Orbiculoidea caneyana and $\underline{O}$. capuliformis in Mexico and, along with $\underline{O}$. missouriensis, highlight the paleobiogeographic relationship of the carboniferous faunas of Nochixtlán with coeval faunas described in the east-central region of the United States.
\end{abstract}

Keywords: Brachiopods, Orbiculoidea, Carboniferous, Chesterian, Morrowan-Desmoinesian, Mexico. 


\section{Introducción}

Las rocas carboníferas que afloran en el área de Santiago Ixtaltepec, en el Municipio de Nochixtlán, Oaxaca, se distinguen por contener distintas asociaciones fósiles que representan comunidades marinas cuya composición taxonómica difiere de acuerdo a la edad y a variaciones de las condiciones paleoambientales bajo las que se desarrollaron. Desde su descubrimiento, sobre las localidades fosilíferas se han realizado trabajos que han tenido como objetivos describir sistemáticamente a los diferentes taxa que se encuentran en cada asociación, establecer las edades de los diferentes niveles estratigráficos y establecer el marco paleoambiental y paleogeográfico en que se acumuló la sucesión de rocas carboníferas de la región. Como parte de estos estudios, para la Formación Ixtaltepec se han descrito diferentes grupos de conuláridos (Escalante-Ruiz et al., 2014), bivalvos (Quiroz-Barroso, 1995; QuirozBarroso y Perrilliat, 1997, 1998), trilobites (Morón-Ríos y Perrilliat, 1988), briozoos (González-Mora y Sour-Tovar, 2014), representantes de diversos ordenes de braquiópodos (Sour-Tovar y Quiroz-Barroso, 1989; Sour-Tovar, 1994; Sour-Tovar y Martínez-Chacón, 2004; Torres-Martínez et al., 2008; Torres-Martínez y Sour-Tovar, 2012; TorresMartínez, 2014; Torres-Martínez y Sour-Tovar, 2016) y crinoideos (Villanueva-Olea et al., 2011; Villanueva-Olea y Sour-Tovar, 2015). Particularmente los braquiópodos son los invertebrados más diversos y abundantes de la sucesión carbonífera de la región oaxaqueña, los cuales representan prácticamente todos los órdenes que existieron durante el Carbonífero (Torres-Martínez, 2014), incluyendo a los del Orden Lingulida que se describen en este trabajo y que forman parte de diferentes asociaciones. Su descripción complementa la lista de los taxa carboníferos presentes en la Formación Ixtaltepec y genera información útil para interpretar las condiciones paleoambientales bajo las cuales se depositó la unidad.

\section{2. Área de estudio}

\subsection{Ubicación Geográfica}

Los especímenes descritos en este trabajo fueron recolectados en la Formación Ixtaltepec, en su sección tipo del Arroyo las Pulgas, que aflora entre las coordenadas $17^{\circ} 31^{\prime}-17^{\circ} 33^{\prime} \mathrm{N}$ y $97^{\circ} 06^{\prime}-97^{\circ} 07^{\prime} \mathrm{W}$, aproximadamente a $500 \mathrm{~m}$ al norte del poblado de Santiago Ixtaltepec, Municipio de Nochixtlán, Oaxaca (Figura 1).

\subsection{Estratigrafía}

La estratigrafía del área (Figura 2) que ha sido descrita en diversos trabajos (p.e. Pantoja-Alor, 1970; QuirozBarroso y Perrilliat, 1997, 1998; Navarro-Santillán et al., 2002; Villanueva-Olea et al., 2011; TorresMartínez y Sour-Tovar, 2012, 2016) se caracteriza por la presencia de rocas sedimentarias del Paleozoico que sobreyacen discordantemente sobre las rocas metamórficas neoproterozoicas del Complejo Oaxaqueño (Solari et al., 2003). Las rocas paleozoicas se han agrupado en la Formación Tiñú, del Cámbrico-Ordovícico (PantojaAlor, 1970; Sour-Tovar y Buitrón, 1987), la formación Santiago, unidad informal del Misisípico Temprano

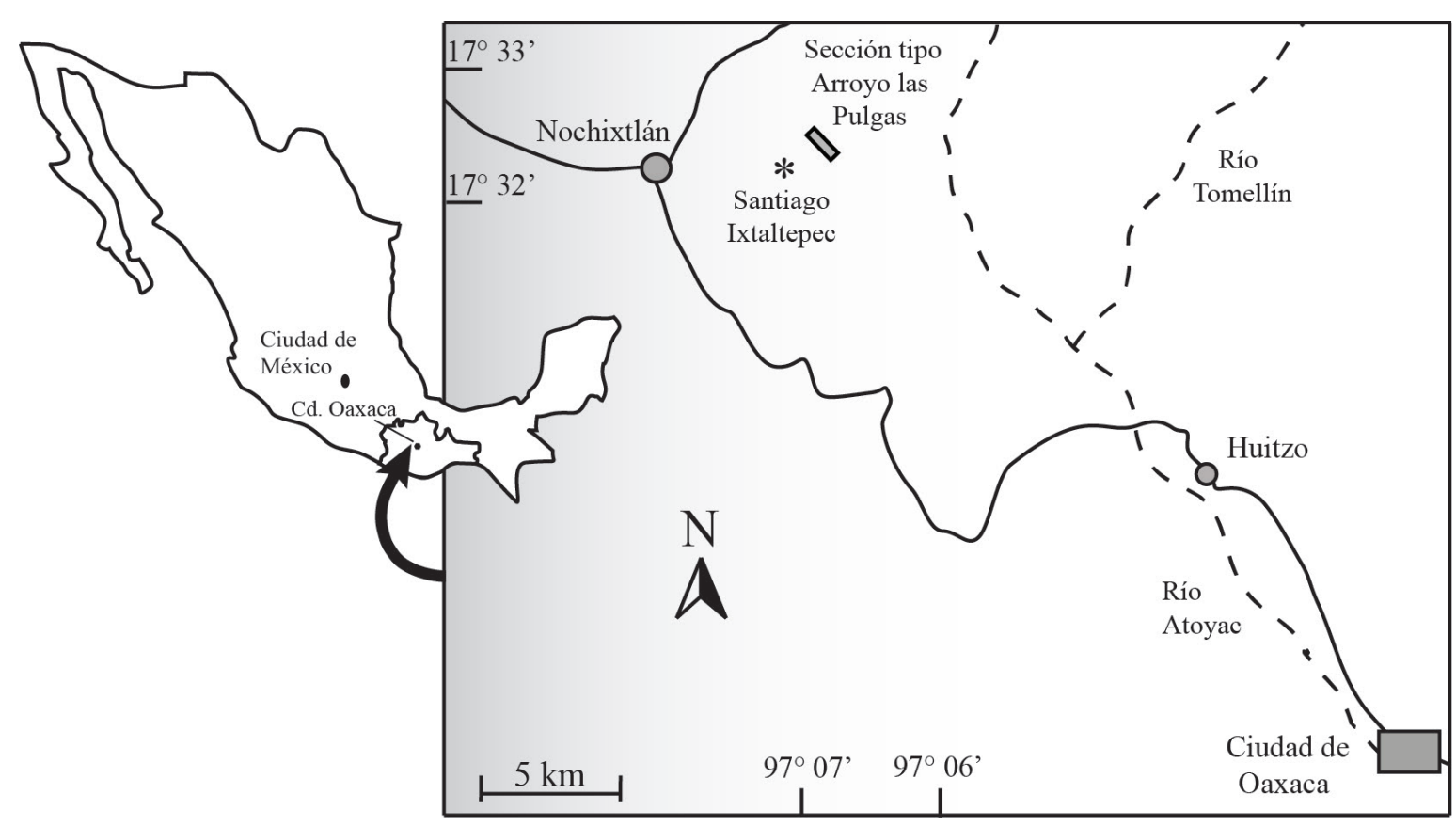

Figura 1. Ubicación geográfica de la Formación Ixtaltepec, sección tipo Arroyo las Pulgas. 


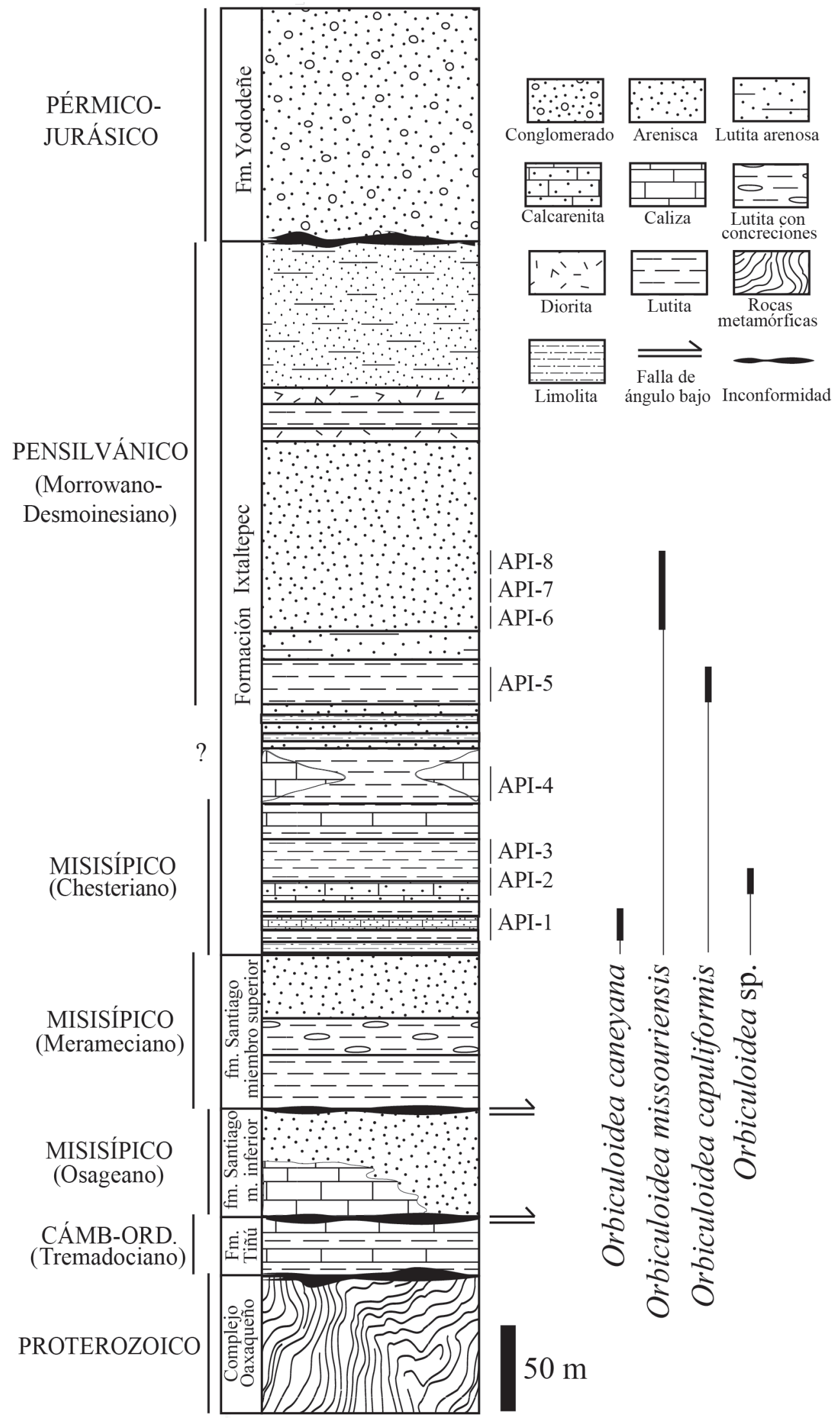

Figura 2. Columna estratigráfica de la sección tipo Arroyo las Pulgas. Se señala la ubicación de cada uno de los niveles fosilíferos de la Formación Ixtaltepec (API-1 a API-8) y los alcances estratigráficos de los taxa descritos en este trabajo. 
(Osageano-Merameciano) (Quiroz-Barroso et al., 2000; Navarro-Santillán et al., 2002; Castillo-Espinoza, 2013) y la Formación Ixtaltepec, con rocas de edades que abarcan desde el Misisípico Tardío (Chesteriano) hasta el Pensilvánico Medio (Morrowano-Desmoinesiano) (SourTovar y Martínez-Chacón, 2004; Torres-Martínez et al., 2008; Torres-Martínez y Sour-Tovar, 2012; Villanueva-Olea y Sour-Tovar, 2015; Torres-Martínez y Sour-Tovar, 2016).

La sección tipo de la Formación Ixtaltepec, unidad en la que se encontraron los orbiculoideos que se describen en este trabajo, presenta un espesor de $430 \mathrm{~m}$, en su base inicia con limolita, seguida de intercalaciones de lutita y calcarenita; por encima se depositan capas delgadas de calcarenita ligeramente arcillosa con bancos gruesos de lutita arenosa de colores grisáceos e intercalaciones de arenisca de grano fino. A partir de la parte media se presentan estratos de limolita y arenisca micácea de grano fino, bancos gruesos de lutita, lutita arenosa y arenisca de grano fino. En el último tercio de la sección se observan intrusiones de diorita a manera de estratos intercalados con lutita arenosa y lutita. Informalmente, para el análisis paleontológico de la formación se han marcado ocho niveles de estudio, nominados con el acrónimo API (Arroyo las Pulgas, Ixtaltepec) y su número correspondiente (API-1 a API-8). Cada nivel presenta características litológicas particulares y contiene una asociación fósil distintiva en todo su espesor.

La sucesión paleozoica está cubierta discordantemente por el conglomerado de la Formación Yododeñe, compuesto de clastos de caliza en los que se han encontrado fusulínidos del Pérmico-Jurásico (Flores de Dios et al., 2000). Sobre esta formación se presentan rocas calcáreas cretácicas (Pantoja-Alor, 1970).

\section{Paleontología Sistemática}

Los ejemplares descritos están depositados en el Museo de Paleontología de la Facultad de Ciencias de la Universidad Nacional Autónoma de México y se encuentran catalogados con las siglas FCMP y número correspondiente. La clasificación y los términos morfológicos utilizados son los referidos por Holmer y Popov (2000, 2007).

Phylum Brachiopoda Duméril, 1805

Subphylum Linguliformea Williams, Carlson, Brunton, Holmer y Popov, 1996

Clase Lingulata Gorjansky y Popov, 1985 Orden Lingulida Waagen, 1885

Superfamilia Discinoidea Gray, 1840

Familia Discinidae Gray, 1840

Género Orbiculoidea d'Orbigny, 1847

Diagnosis. Concha circular de fuertemente dorsibiconvexa a convexo-plana; ornamentación de ambas valvas usualmente bien desarrollada, con liras concéntricas; valva dorsal de cónica a subcónica, ápice variablemente posicionado; valva ventral de subcónica baja a ligeramente cóncava, cicatriz del pedicelo estrecha, cerrada anteriormente por un listrium; foramen en la terminación posterior al listrium, formando una cámara interna que se abre por delante del margen posterior (Holmer y Popov, 2000).

Ocurrencia. Orbiculoidea es un género de distribución cosmopolita cuyo rango estratigráfico se extiende desde el Silúrico hasta el Pérmico (Holmer y Popov, 2000).

Especie tipo. Orbicula forbesii Davidson, 1848. Wenlock, Inglaterra. Silúrico.

\section{Orbiculoidea caneyana (Girty, 1909)}

Figuras 3.1, 3.2

Lingulidiscina newberryi var. caneyana Girty, 1909: 19-20, lám. 1, figs. 13-17.

Lingulidiscina newberryi var. caneyana Girty, 1911: 40-41, lám. 2, figs. 4, 5.

Lingulidiscina newberryi caneyana (Girty). Morgan, 1924: lám. 43, fig. 7.

Orbiculoidea newberryi var. caneyana (Girty). Girty, 1929: 78.

Lingulidiscina newberryi caneyana (Girty). Elias y Branson, 1959: 8.

Orbiculoidea caneyana (Girty). McKnight y Fischer, 1970: 59.

Especímenes. Dos valvas dorsales (FCMP 1154, 1155) $\mathrm{y}$ dos valvas ventrales (FCMP 1156, 1157).

Descripción. Concha muy pequeña, circular en línea externa, convexo-plana; el ejemplar de mayor tamaño mide $6.2 \mathrm{~mm}$ de largo por $6.1 \mathrm{~mm}$ de ancho. La valva dorsal es cónica, de convexidad muy baja; el ápice se ubica a dos tercios de diámetro del margen posterior de la valva; ligeramente convexa en pendiente posterior al ápice, al igual que en la pendiente anterior a éste; pendientes laterales ligeramente convexas. La valva ventral es semiplana, con el ápice subcentral; cicatriz del pedicelo estrecha, ligeramente deprimida. La superficie de ambas valvas está marcada por numerosas liras equidistantes, finas, a una distancia de un milímetro del centro de la valva de cinco a siete ocupan un espacio de $1 \mathrm{~mm}$; interespacios aplanados.

Ocurrencia. Formación Ixtaltepec, sección tipo Arroyo las Pulgas, nivel API-1.

Discusión. Orbiculoidea caneyana se ha reportado para el Misisípico Tardío de los Estados Unidos en Oklahoma (Girty, 1909), Arkansas (Girty, 1911) y Kansas (McKnight y Fischer, 1970). O. caneyana se puede distinguir de especies contemporáneas y con cierta similitud morfológica, como Orbiculoidea newberryi marshallensis y Orbiculoidea moorefieldana de Arkansas (Girty, 1911) y Orbiculoidea newberryi ovata de Oklahoma y Arkansas (Girty, 1909; 1911) por su concha más pequeña, con una valva dorsal de menor convexidad y un ápice ubicado a dos tercios de diámetro del margen posterior. Este es el primer reporte de la especie para México. 
Orbiculoidea missouriensis (Shumard y Swallow, 1858)

Figuras 3.3-3.14

Discina missouriensis Shumard y Swallow, 1858: 221. Discina manhattanensis Meek y Hayden, 1859: 25.

Orbiculoidea manhattanensis (Meek y Hayden). Hall y Clarke, 1892: lám. 4, fig. 20.

Orbiculoidea missouriensis (Shumard y Swallow). Dunbar y Condra, 1932: 42-45, lám. 1, figs. 12-17.

Orbiculoidea missouriensis (Shumard y Swallow). Hoare, 1961: 22, 23, lám.1, figs. 3-5.

Orbiculoidea missouriensis (Shumard y Swallow). Sturgeon y Hoare, 1968: 22, lám. 1, figs. 7-11.

Orbiculoidea missouriensis (Shumard y Swallow). Kues, 1984: 109.

Especímenes. Cinco valvas dorsales (FCMP 1158-1162) y siete valvas ventrales (FCMP 1163-1169).

Descripción. Concha pequeña, subcircular en línea externa, biconvexa a convexo-plana; el ejemplar de mayor tamaño mide $17.7 \mathrm{~mm}$ de largo por $15.9 \mathrm{~mm}$ de ancho. La valva dorsal es cónica; ápice incurvado, localizado a un tercio de diámetro del margen posterior de la valva; la pendiente posterior del ápice es ligeramente cóncava, mientras que la pendiente anterior es ligeramente convexa; pendientes laterales ligeramente convexas. La valva ventral es resupinada o ligeramente aplanada; ápice subcentral; cicatriz del pedicelo estrecha, termina en la apertura frontal del margen posterior. La superficie de la valva dorsal presenta numerosas liras, separadas entre ellas de manera irregular, finas, a una distancia de tres milímetros del centro de la valva cuatro o cinco ocupan un espacio de $1 \mathrm{~mm}$; liras radiales finas atraviesan las liras concéntricas y sus interespacios; la superficie de la valva ventral presenta liras con una separación regular.

Ocurrencia. Formación Ixtaltepec, sección tipo Arroyo las Pulgas, niveles API-6, API-7 y API-8.

Discusión. Los ejemplares de Orbiculoidea missouriensis de la Formación Ixtaltepec se caracterizan por la presencia de una valva dorsal cónica, con un ápice localizado a un tercio de diámetro del margen posterior y una valva ventral resupinada o ligeramente aplanada con un ápice subcentral, todos rasgos previamente descritos por Dunbar y Condra (1932, p. 42) y Hoare (1961, p. 23, lám. 1, figs. 3-5) para la especie. Orbiculoidea missouriensis ha sido reportada para el Pensilvánico de gran parte del territorio de los Estados Unidos, por ejemplo para Misuri, Kansas (Shumard y Swallow, 1858; Meek y Hayden, 1859; Hoare, 1961), Nebraska, Illinois (Dunbar y Condra, 1932), Ohio (Sturgeon y Hoare, 1968) y Nuevo México (Kues, 1984). Previamente había sido referida para el área de Santiago Ixtaltepec (SourTovar, 1994), confirmando con este trabajo la presencia de la especie en la región y para México.

Orbiculoidea capuliformis (McChesney, 1860)

Figuras 3.15-3.20
Discina capuliformis McChesney, 1860: 72.

Discina convexa White, 1884: 121, lám. 25, fig. 9.

Discina convexa White. Beede, 1900: 55, lám. 8, figs. $3,3 b$.

Orbiculoidea capuliformis (McChesney). Morningstar, 1922: 170, 171.

Orbiculoidea capuliformis (McChesney). Dunbar y Condra, 1932: 46, 47, lám. 1, figs. 20-22.

Orbiculoidea capuliformis (McChesney). Hoare, 1961: 23, 24, lám. 1, figs. 8, 9.

Orbiculoidea capuliformis (McChesney). Sturgeon y Hoare, 1968: 22, lám. 1, figs. 12-15.

Orbiculoidea capuliformis (McChesney). Kues, 1984: 109.

Especímenes. Tres valvas dorsales (FCMP 1170-1172) $\mathrm{y}$ tres valvas ventrales (FCMP 1173-1175).

Descripción. Concha pequeña a mediana, subcircular a suboval en línea externa, biconvexa a convexo-plana, el ejemplar de mayor tamaño mide $23.6 \mathrm{~mm}$ de largo por 20.3 $\mathrm{mm}$ de ancho. La valva dorsal es subcónica; ápice incurvado, se ubica a un cuarto de diámetro del margen posterior de la valva; la pendiente posterior del ápice es ligeramente cóncava, la pendiente anterior es ligeramente convexa; pendientes laterales ligeramente convexas. La valva ventral es ligeramente convexa o plana; ápice ubicado posterior al centro de la valva; cicatriz del pedicelo elongada, ovoide, termina en el margen posterior. La superficie de ambas valvas presenta numerosas liras finas, equidistantes, a una distancia de tres milímetros del centro de la valva tres o cuatro ocupan un espacio de $1 \mathrm{~mm}$; numerosas liras radiales finas atraviesan las liras concéntricas y sus interespacios.

Ocurrencia. Formación Ixtaltepec, sección tipo Arroyo las Pulgas, nivel API-5.

Discusión. La presencia en el material de Nochixtlán de una valva dorsal subcónica, con un ápice incurvado a un cuarto de diámetro del margen posterior y una pendiente anterior ligeramente convexa, una valva ventral ligeramente convexa (o plana con el ápice al centro) y una cicatriz del pedicelo ovoide, coinciden con los rasgos descritos por Hoare (1961, p. 23, 24, lám. 1, figs. 8, 9) para ejemplares de O. capuliformis (McChesney) del Desmoinesiano de Kansas; sin embargo el material de Santiago Ixtaltepec presenta una elongación ligeramente mayor al material descrito para Kansas. La especie de O. capuliformis ha sido reportada para el Pensilvánico Medio de los Estados Unidos en Misuri, Illinois, Nebraska (Dunbar y Condra, 1932), Kansas (Hoare, 1961), Ohio (Sturgeon y Hoare, 1968) y Nuevo México (Kues, 1984). Los ejemplares aquí descritos constituyen el primer registro para el territorio mexicano.

\section{Orbiculoidea sp.}

Figura 3.21

Especímenes. Un molde externo de valva dorsal (FCMP 1176). 

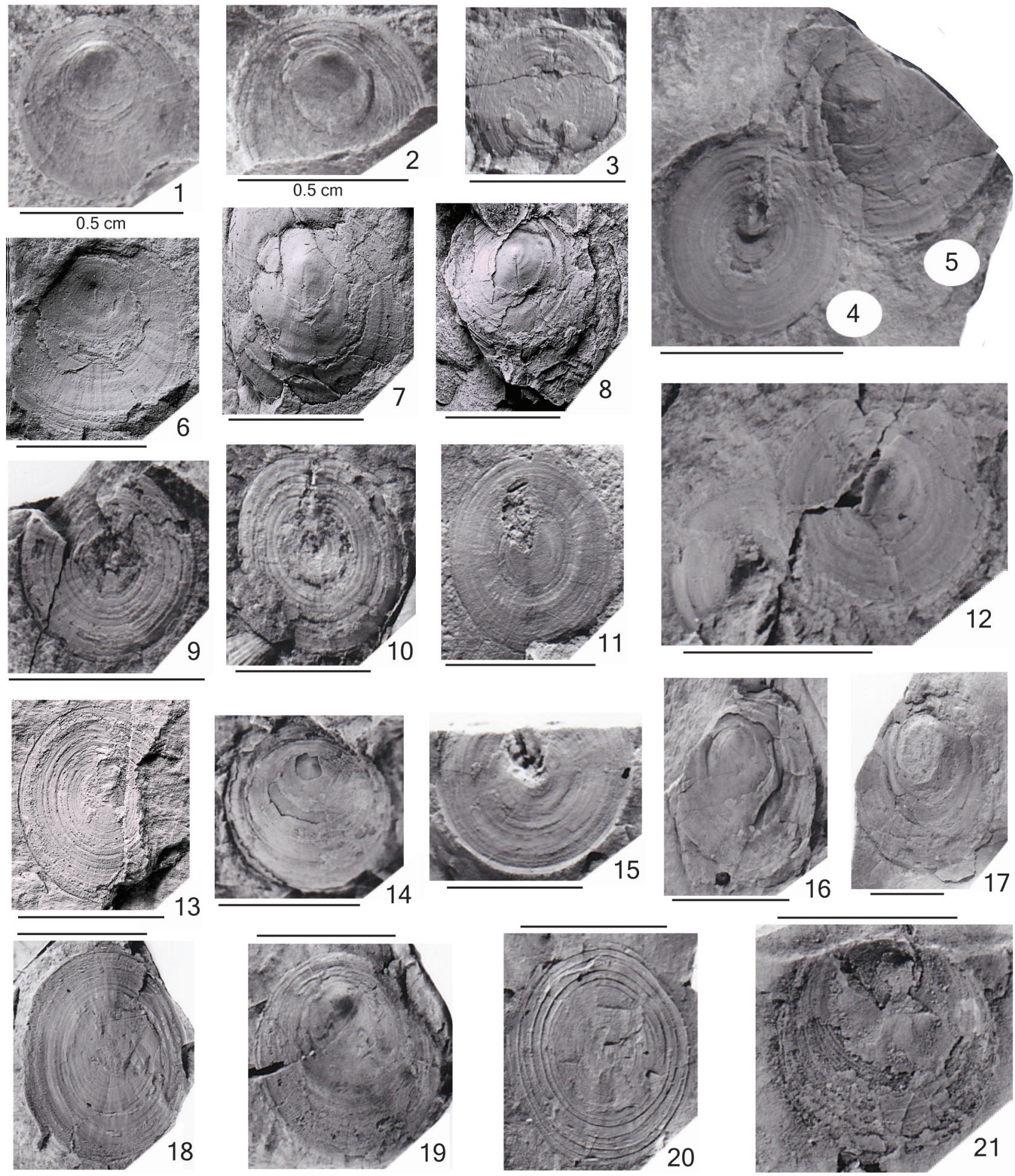

Figura 3. (1, 2) Orbiculoidea caneyana (Girty, 1909): 1, valva dorsal, interior, FCMP 1154; 2, valva dorsal, interior, FCMP 1155. (3-14) Orbiculoidea missouriensis (Shumard y Swallow, 1858): 3, valva ventral, interior, FCMP 1163; 4, valva ventral, interior, FCMP 1164; 5, valva dorsal, exterior, FCMP 1158; 6, valva dorsal, interior, FCMP 1159; 7, valva dorsal, exterior, FCMP 1160; 8, valva dorsal, exterior, FCMP 1161; 9 , valva ventral, exterior, FCMP 1165; 10, valva ventral, interior, FCMP 1166; 11, valva ventral, interior, FCMP 1167; 12, valva ventral, interior, FCMP 1168; 13, valva ventral, interior, FCMP 1169; valva dorsal, exterior, FCMP 1162. (15-20) Orbiculoidea capuliformis (McChesney, 1860): 15, valva ventral, interior, FCMP 1173; 16, valva dorsal, exterior, FCMP 1170; 17, valva dorsal, exterior, FCMP 1171; 18, valva ventral, exterior, FCMP 1174; 19, valva dorsal, interior, FCMP 1172; 20, valva ventral, exterior, FCMP 1175. (21) Orbiculoidea sp.: 21, valva dorsal, interior, FCMP 1176. Escala a $1 \mathrm{~cm}$. 
Descripción. Valva dorsal pequeña, subcircular en línea externa, convexidad muy baja, midiendo $12.3 \mathrm{~mm}$ de largo por $13.6 \mathrm{~mm}$ de ancho; ápice corto, ligeramente curvo, se ubica a un octavo de diámetro del margen posterior de la valva; las pendientes posterior y anterior del ápice son ligeramente convexas; pendientes laterales semiplanas. En la superficie se presentan numerosas liras finas, equidistantes, a una distancia de tres milímetros del centro de la valva tres liras ocupan un espacio de $1 \mathrm{~mm}$; numerosas liras radiales finas atraviesan las liras concéntricas y sus interespacios.

Ocurrencia. Formación Ixtaltepec, sección tipo Arroyo las Pulgas, nivel API-2.

Discusión. Los rasgos morfológicos del ejemplar que se describe permiten asignarlo de manera fiable al género Orbiculoidea; sin embargo, lo escaso y fragmentario del material disponible impide su asignación específica. El ejemplar descrito se distingue de las otras especies encontradas en la Formación Ixtaltepec por su valva dorsal poco convexa, la ubicación del ápice, que es corto, a un octavo de diámetro del margen posterior y por la disposición de las liras.

\section{Discusión}

\subsection{Consideraciones bioestratigráficas y paleobiogeográficas}

De las especies de discínidos que se describen en el presente trabajo, Orbiculoidea caneyana y Orbiculoidea sp. se hallaron respectivamente en los niveles API-1 y API-2 de la Formación Ixtaltepec, en los que también se encuentran, entre otros taxa, los braquiópodos Semicostella sp., Inflatia inflata, Keokukia sp. (Torres-Martínez y Sour-Tovar, 2016), Echinoconchella elegans, Marginovatia minor, Ovatia muralis y Sinuatella sp. (Torres-Martínez y Sour-Tovar, 2012) y el crinoideo Lomalegnum hormidium (VillanuevaOlea et al., 2011), taxa considerados índice del Chesteriano (Misisípico Tardío). Previamente O. caneyana sólo había sido referida para localidades del Chesteriano de Arkansas, Oklahoma y Kansas (Girty, 1909, 1911; McKnight y Fischer, 1970) y su presencia en Oaxaca apoya la datación que se ha postulado para los niveles API-1 al API-3 de la Formación Ixtaltepec. La tercera especie de discínido, Orbiculoidea capuliformis, se encontró en el nivel API-5 y Orbiculoidea missouriensis en los niveles API-6, API-7 y API-8. Estas dos especies descritas originalmente para Misuri, Illinois y Nebraska, son consideradas típicamente pensilvánicas (Dunbar y Condra, 1932; Hoare, 1961; Sturgeon y Hoare, 1968) y su asociación con los braquiópodos Neochonetes granulifer (Sour-Tovar y Martínez-Chacón, 2004), Reticulatia huecoensis, Buxtonia websteri (Torres-Martínez y Sour-Tovar, 2016), Echinaria knighti, Linoproductus prattenianus, Linoproductus platyumbonus, Marginovatia pumila, Marginovatia aureocollis (Torres-Martínez y Sour-Tovar, 2012) y Neospirifer dunbari (Torres-Martínez et al., 2008) corroboran la edad pensilvánica de los estratos portadores.

Las especies de Orbiculoidea que se describen en el presente trabajo han sido referidas como formas comunes en diferentes localidades del Carbonífero de los Estados Unidos, en particular de la región centro-este conocida como el Midcontinent (p.e. Arkansas, Oklahoma, Misuri, Kansas, Nebraska, Illinois y Nuevo México). Su presencia en las localidades de Santiago Ixtaltepec remarca la afinidad de ambas faunas señalada en trabajos previos, por ejemplo, con el estudio de bivalvos carboníferos de la Formación Ixtaltepec (Quiroz-Barroso y Perrilliat, 1997, 1998; QuirozBarroso et al., 2000), braquiópodos osageanos del miembro inferior de la formación Santiago (Navarro-Santillán et al., 2002), diferentes grupos de braquiópodos del Carbonífero medio (Sour-Tovar y Martínez-Chacón, 2004; TorresMartínez et al., 2008; Torres-Martínez y Sour-Tovar, 2012, 2016) y crinoideos (Villanueva-Olea et al., 2011; Villanueva-Olea y Sour-Tovar, 2015).

\subsection{Implicaciones paleoecológicas}

En todos los niveles en que se han encontrado discínidos, estos braquiópodos se encuentran representados por valvas desarticuladas, preservados duripárticamente o como moldes internos autigénicos; todas las valvas se encontraron en posición horizontal sin una dirección específica, aisladas, sin señales de bioturbación, transporte, disolución o abrasión y ello permite inferir que los ejemplares se acumularon en ambientes de depósito con un nivel energético de bajo a medio, sufriendo un enterramiento rápido sin un transporte previo. La desarticulación que se observa en todos los ejemplares de orbiculoideos de Ixtaltepec se explica por el tipo de articulación que presentan estos braquiópodos ya que, como cualquier otro linguliformeo, carecen de una línea de charnela y de mecanismos articuladores rígidos (Álvarez y Martínez-Chacón, 2009), lo que resulta en una rápida desarticulación de las valvas posterior a la muerte del animal, aún cuando las condiciones energéticas sean bajas. Los mismos rasgos tafonómicos sugieren que los braquiópodos estudiados, a diferencia de la mayoría de los discínidos que se encuentran en aglomeraciones de varios individuos dispuestos unos sobre otros (Mergl, 2010), fueron formas solitarias.

La región en que se depositaron las rocas carboníferas presentes en Santiago Ixtaltepec estuvo sometida a diversos cambios tectónicos durante el Carbonífero, que modificaron drásticamente el medio marino e influyeron en la estructura de las diferentes comunidades de invertebrados bentónicos del lugar. La interpretación y caracterización puntual de tales cambios aún se encuentran en desarrollo, habiéndose caracterizado solamente los paleoambientes de algunos de los niveles estratigráficos que conforman la sucesión carbonífera de la región. Como se describe en las consideraciones bioestratigráficas, los ejemplares de orbiculoideos descritos en este trabajo fueron recolectados en distintos niveles fosilíferos de la Formación Ixtaltepec; 
niveles que han sido relacionados con diferentes ambientes sedimentarios de acuerdo al tipo de fauna y litología que presentan las rocas portadoras (Quiroz-Barroso y Perrilliat, 1997, 1998; González-Mora y Sour-Tovar, 2014; Villanueva-Olea y Sour-Tovar, 2015). Cada especie de braquiópodo inarticulado se puede relacionar directamente con un ambiente en particular dependiendo del nivel fosilífero en el que se encontró: API-1 y API-2 (arrecife), API-3 (prodelta), API-5 (nerítico somero), API-6 y API-7 (peri-arrecifal) o API-8 (plataforma externa o de offshore). Los estratos correspondientes a la unidad API-4 sólo presentan fósiles traza que aún se encuentran en estudio con la finalidad de inferir el paleoambiente en que se depositó esta unidad. Es importante resaltar que aunque se trataba de ambientes diferentes, todos presentaban condiciones de aguas poco profundas, buena luminosidad, oxigenación y energía media-baja (Torres-Martínez, 2014) (Tabla 1).

Al igual que en este trabajo, en otras publicaciones Orbiculoidea capuliformis ha sido referida como especie presente en depósitos de ambientes neríticos y Orbiculoidea missouriensis de ambientes de tipo peri-arrecifal y de plataforma externa (offshore) (Shumard y Swallow, 1858; Meek y Hayden, 1859; Kues, 1984). En el caso de Orbiculoidea caneyana, este el primer reporte donde se le relaciona a un ambiente en particular, específicamente al de tipo arrecifal.

\section{Agradecimientos}

Los autores agradecen los valiosos comentarios y observaciones hechas por los árbitros Gabriela A. Cisterna y Carlos Esquivel-Macías; el apoyo proporcionado por D. Navarro-Santillán en el trabajo de campo y el proceso curatorial, y a H. Hernández-Campos y E.P. Porras-López por la elaboración del material fotográfico. Este trabajo es parte de la tesis doctoral de M.A. Torres-Martínez titulada "Braquiópodos carboníferos del área de Santiago Ixtaltepec, Oaxaca. Implicaciones paleoambientales, estratigráficas y paleobiogeográficas" apoyada por el CONACyT con la subvención número 174854 y por la Dirección General de Asuntos del Personal Académico (DGAPA), UNAM a través del proyecto PAPIIT IN215013.

\section{Referencias}

Álvarez, F., Martínez-Chacón, M.L., 2009, Braquiópodos, en MartínezChacón M.L., Rivas, P. (eds.), Paleontología de invertebrados: Oviedo, Ediciones de la Universidad de Oviedo, 377-418.

Beede, J.W., 1900, Carboniferous invertebrates: Kansas Geological Survey, 6, $187 \mathrm{p}$.

Castillo-Espinoza, K.M., 2013, Sistemática de braquiópodos, cefalópodos y crinoideos del Misisípico medio de la formación Santiago, Santiago Ixtaltepec, Oaxaca: México D.F., Universidad Nacional Autónoma de México, Tesis de Maestría, 112 p.

Davidson, T., 1848, Mémoire sur les Brachiopodes du Système Silurien supérieur de l'Angleterre: Société Géologique de France, Bulletin
Tabla 1. Paleoambientes señalados para cada nivel estratigráfico donde se recolectaron los orbiculoideos de la Formación Ixtaltepec.

\begin{tabular}{lcc}
\hline \multicolumn{1}{c}{ Especie } & $\begin{array}{c}\text { Nivel } \\
\text { fosilífero }\end{array}$ & Paleoambiente \\
\hline $\begin{array}{l}\text { Orbiculoidea caneyana } \\
\text { Orbiculoidea } \text { sp. }\end{array}$ & API-1 & Arrecifal \\
\multicolumn{1}{c}{ API-2 } & Arrecifal \\
---------- & API-3 & Prodelta \\
Orbiculoidea capuliformis & API-4 & $? ? ?$ \\
Orbiculoidea missouriensis & API-6 & Nerítico somero \\
Orbiculoidea missouriensis & API-7 & Peri-arrecifal \\
Orbiculoidea missouriensis & API-8 & Plataforma externa \\
\hline
\end{tabular}

(séries 2), 5, 309-338, 370-374 p.

Duméril, A.M.C., 1805, Zoologie analytique, ou méthode naturelle de classification des animaux, rendue plus facile à l'aide de table aux synoptiques: Allais, Paris. xxiv +344 p.

Dunbar, C.O., Condra, G.E., 1932, Brachiopoda of the Pennsylvanian system in Nebraska: Nebraska Geological Survey, Bulletin 5, Second Series, $377 \mathrm{p}$.

Elias, M.K., Branson, C.C., 1959, Type section of the Caney Shale: Oklahoma Geological Survey, 52, $24 \mathrm{p}$.

Escalante-Ruíz, A.R., Quiroz-Barroso, S.A., Sour-Tovar, F., 2014, Conuláridos misisípicos de Nochixtlán, Oaxaca, México: consideraciones sistemáticas, estratigráficas y paleobiogeográficas: Revista Brasileira de Paleontología, 17(2), 195-206.

Flores de Dios, A., Vachard, D., Buitrón, B.E., 2000, La cubierta sedimentaria pérmica superior (Formaciones Olinalá, Ihualtepec y Yododeñe) de los terrenos Zapoteco y Mixteco, evolución sedimentológica e interpretaciones paleogeográficas (resumen), en 2a. Reunión Nacional de Ciencias de la Tierra, Puerto Vallarta, Jal., México, Unión Geofísica Mexicana, GEOS 20, 324-325.

Girty, G.H., 1909, The fauna of the Caney Shale of Oklahoma: Bulletin United States of Geological Survey, 377, $106 \mathrm{p}$.

Girty, G.H., 1911, The fauna of the Moorefield Shale of Arkansas: Bulletin United States of Geological Survey, 439, $148 \mathrm{p}$.

Girty, G.H., 1929, The fauna of the Middle Boone near Batesville, Arkansas: Professional Paper United States of Geological Survey, 154-B, 73-103.

González-Mora, S., Sour-Tovar, F., 2014, Briozoarios del Orden Fenestrida, Pensilvánico de la Formación Ixtaltepec, Municipio de Nochixtlán, Oaxaca; consideraciones paleoambientales: Boletín de la Sociedad Geológica Mexicana, 66(3), 471-482.

Gorjansky, V.Iu. [Gorjansky, V.Y., Gorjansky, W.J.], Popov, L.E., 1985, Morfologiya, sistematicheskoe polozhenie i proiskhozhdenia bezzamkovykh brakhiopod s karbonatnoj rakovinoj. Paleontologicheskii Zhurnal, 1985(3), 3-14.

Gray, J.E., 1840, Synopsis of the Contents of the British Museum: London, British Museum, 42nd edition, $370 \mathrm{p}$.

Hall, J., Clarke, J.M., 1892, An Introduction to the Study of the Genera of Palaeozoic Brachiopoda: New York, Charles van Benthuysen \& Sons, $367 \mathrm{p}$

Hoare, R.D., 1961, Desmoinesian Brachiopoda and Mollusca from southwest Missouri: Missouri, University of Missouri press, 263 p.

Holmer, L.E., Popov L.E., 2000, Lingulida, en Kaesler, R.L. (ed.), Treatise on Invertebrate Paleontology, Part H, Brachiopoda, Revised, Volume 2: Kansas, The Geological Society of America, Inc. and the University of Kansas, Boulder, Colorado and Lawrence press, 32-97.

Holmer, L.E., Popov, L.E., 2007, Lingulida, en Selden, P.A. (ed.), Treatise on Invertebrate Paleontology Part H, Brachiopoda, Revised, Volume 6 - Supplement: Kansas, The Geological Society of America, Inc. and the University of Kansas, Boulder, Colorado and Lawrence press, 2532-2559. 
Kues, B.S., 1984, Pennsylvanian stratigraphy and paleontology of the Taos area, north-central New Mexico, Río Grande Rift, Northern New Mexico: New Mexico Geological Society Guidebook, 35, 107-114.

McChesney, J.H., 1860, Descriptions of new species of fossils from the Paleozoic rocks of the western states: Chicago, Extract Transactions of the Chicago Academy of Sciences, $76 \mathrm{p}$.

McKnight, E.T., Fischer, R.P., 1970, Geology and ore deposits of the Picherfield, Oklahoma and Kansas: Professional Paper United States of Geological Survey 588, $165 \mathrm{p}$.

Meek, F.B., Hayden, F.V., 1859, Geological explorations in Kansas territory: Proceedings of the Academy of Natural Science of Philadelphia, 256-266.

Mergl, M., 2010, Discinid brachiopod life assemblages, fossil and extant: Bulletin of Geosciences, 85(1), 27-38.

Morgan, G.D., 1924, Geology of the Stonewall quadrangle, Oklahoma: Oklahoma Bulletin Bureau Geology, 2, 248 p.

Morningstar, H., 1922, Pottsville fauna of Ohio: Ohio Geological Survey Bulletin, 25, $312 \mathrm{p}$.

Morón-Ríos, A., Perrilliat, M.C., 1988, Una especie nueva del género Griffithides Portlock (Arthropoda, Trilobita) del Paleozoico Superior de Oaxaca: Revista del Instituto de Geología, Universidad Nacional Autónoma de México, 7, 67-70.

Navarro-Santillán, D., Sour-Tovar, F., Centeno-García, E., 2002, Lower Mississippian (Osagean) brachiopods from the Santiago Formation, Oaxaca, Mexico: stratigraphic and tectonic implications: Journal of South American Earth Sciences, 15(3), 327-336.

d'Orbigny, A., 1847, Considérations zoologiques et géologiques sur les brachiopodes ou palliobranches, parts 1-2: Comptes Rendus Hebdomadaires des Séances de l'Académie des Sciences, 25(5), 193-195; 25(7), 266-269.

Pantoja-Alor, J., 1970, Rocas sedimentarias paleozoicas de la región centro-septentrional de Oaxaca, en Segura, L.R., Rodríguez T.R., (eds.), Libro guía de la excursión México-Oaxaca: México, Sociedad Geológica Mexicana, 67-84

Quiroz-Barroso, S.A., 1995, Bivalvos del Carbonífero de Nochixtlán, Oaxaca: México D.F., Universidad Nacional Autónoma de México, Tesis Doctoral, $86 \mathrm{p}$.

Quiroz-Barroso, S.A., Perrilliat, M.C., 1997, Pennsylvanian Nuculoids (Bivalvia) from the Ixtaltepec Formation, Oaxaca, Mexico: Journal of Paleontology, 71(3), 400-407.

Quiroz-Barroso, S.A., Perrilliat, M.C., 1998, Pennsylvanian bivalves from the Ixtaltepec Formation, Mexico: Journal of Paleontology, 72(6), 1011-1024.

Quiroz-Barroso, S.A., Pojeta Jr., J., Sour-Tovar, F., Morales-Soto, S., 2000, Pseudomulceodens: A Mississippian rostroconch from Mexico: Journal of Paleontology, 74(6), 1184-1186.

Shumard, B.F., Swallow, G.C., 1858, Descriptions of new fossils from the Coal Measures of Missouri and Kansas: Transactions of the Academy of Science of St. Louis, 1, 198-227.

Solari, L.A., Keppie, J.D., Ortega-Gutiérrez, F., Cameron, K.L., Lopez, R., Hames, W.E., 2003, 990 and 1100 Ma Grenvillian tectonothermal events in the northern Oaxacan Complex, southern Mexico: roots of an orogen: Tectonophysics, 365, 257-282.

Sour-Tovar, F., 1994, Braquiópodos pensilvánicos del área de Santiago Ixtaltepec, Municipio de Nochixtlán, Oaxaca: México D.F., Universidad Nacional Autónoma de México, Tesis de Maestría, 55 p.
Sour-Tovar, F., Buitrón, B.E., 1987, Los graptolitos del Tremadociano de Ixtaltepec, Oaxaca. Consideraciones sobre el límite CámbricoOrdovícico de la región: Revista de la Sociedad Mexicana de Paleontología, 1, 380-395.

Sour-Tovar, F., Martínez-Chacón, M.L., 2004, Braquiópodos chonetoideos del Carbonífero de México: Revista Española de Paleontología, 19(2), 125-138.

Sour-Tovar, F., Quiroz-Barroso, S.A., 1989, Braquiópodos pensilvánicos (Strophomenida) de la Formación Ixtaltepec, Santiago Ixtaltepec, Oaxaca: Revista de la Sociedad Mexicana de Paleontología, 2, 5-17.

Sturgeon, M.T., Hoare, R.D., 1968, Pennsylvanian brachiopods of Ohio: Ohio Geological Survey Bulletin, 63, 95 p.

Torres-Martínez, M.A., 2014, Braquiópodos carboníferos del área de Santiago Ixtaltepec, Oaxaca. Implicaciones paleoambientales, estratigráficas y paleobiogeográficas: México D.F., Universidad Nacional Autónoma de México, Tesis Doctoral, 191 p.

Torres-Martínez, M.A., Sour-Tovar, F., 2012, Nuevos braquiópodos prodúctidos (Rhynchonelliformea, Strophomenata) del Carbonífero de la región de Nochixtlán, Oaxaca: Revista Mexicana de Ciencias Geológicas, 29(3), 696-712.

Torres-Martínez, M.A., Sour-Tovar, F., 2016, New productid brachiopods (Productoidea) from the Carboniferous of Ixtaltepec Formation, Oaxaca, Mexico: Journal of Paleontology. (en prensa).

Torres-Martínez, M.A., Sour-Tovar, F., Pérez-Huerta, A., 2008, Neospiriferinid brachiopods (Spiriferida, Trigonotretidae) from Ixtaltepec Formation, Pennsylvanian of Oaxaca State, Southern Mexico: Fossils and Strata, Lethaia Supplement, 54, 157-166.

Villanueva-Olea, R., Sour-Tovar, F., 2015, A new genus and four new species of cladids (Crinoidea, Cladida) from the Carboniferous of Oaxaca, Southern Mexico: Journal of Systematic Palaeontology, 13(7), 527-542

Villanueva-Olea, R., Castillo-Espinoza, K.M., Sour-Tovar, F., QuirozBarroso, S.A., Buitrón, B.E., 2011, Placas columnares de crinoides del Carbonífero de la Región de Santiago Ixtaltepec, Municipio de Nochixtlán, Oaxaca; consideraciones estratigráficas y paleobiogeográficas: Boletín de la Sociedad Geológica Mexicana, 63(3), 429-443

Waagen, W.H., 1885, Salt Range fossils, vol. I, part 4, Productus Limestone fossils, Brachiopoda: Palaeontologia Indica, 13(5), 729-770.

White, C.A., 1884, The fossils of the Indiana rocks: Indiana, U.S.A., Indiana Department in Geology and Natural History, 13th Annual Report, 3, 107-180.

Williams, A., Carlson, S.J., Brunton, C.H.C., Holmer, L.E., Popov, L., 1996, A supra-ordinal classification of the Brachiopoda: Philosophical Transactions of the Royal Society of London, Series B (351), 1171-1193.

Manuscrito recibido: Julio 3, 2015

Manuscrito corregido recibido: Octubre 15, 2015

Manuscrito aceptado: Octubre 22, 2015 